\title{
Association of Systemic Inflammation with Depressive Symptoms in Individuals with COPD
}

\author{
Hilary C Strollo (iD \\ Seyed M Nouraie (D) \\ Karin F Hoth ${ }^{2}$ \\ Craig M Riley ${ }^{3}$ \\ Chad Karoleski ${ }^{1}$ \\ Yingze Zhang' \\ Nicola A Hanania ${ }^{4}$ \\ Russell P Bowler 5,6 \\ Jessica Bon' \\ Frank C Sciurbal \\ 'University of Pittsburgh Medical Center, \\ Department of Medicine, Department of \\ Pulmonary Allergy and Critical Care \\ Medicine, Pittsburgh, PA, USA; \\ ${ }^{2}$ University of lowa Carver College of \\ Medicine, Department of Psychiatry, \\ lowa City, IA, USA; ${ }^{3}$ Chester County \\ Hospital, University of Pennsylvania \\ Health System, West Chester, PA, USA; \\ ${ }^{4}$ Baylor College of Medicine, Department \\ of Pulmonary, Critical Care and Sleep \\ Medicine, Houston, TX, USA; ${ }^{5}$ National \\ Jewish Health, Department of Medicine, \\ Denver, CO, USA; ' ${ }^{6}$ niversity of \\ Colorado School of Medicine, Denver, \\ CO, USA
}

Correspondence: Hilary C Strollo University of Pittsburgh Medical Center, Department of Medicine, Department of Pulmonary Allergy and Critical Care Medicine, NW 628 UPMC Montefiore, 3459 Fifth Avenue, Pittsburgh, PA, 15213, USA

$\mathrm{Tel}+\mid$ 4I 2-692-2210

$\mathrm{Fax}+\mid$ 4|2-692-4842

Email strolloh2@upmc.edu
Rationale: Depression is a prevalent comorbidity of chronic obstructive pulmonary disease (COPD) that, along with COPD, has been associated with inflammation. An association between inflammation and depression in COPD has not been validated in a large COPD cohort.

Methods: Individuals from the University of Pittsburgh SCCOR cohort and the COPDGene cohort with tobacco use history and airway obstruction $\left(\mathrm{FEV}_{1} / \mathrm{FVC}<0.7\right)$ were evaluated using the Beck Depression Inventory II (BDI-II) and the Hospital Anxiety and Depression Scale (HADS), respectively. Participants completed symptom-related questionnaires and plasma IL-6 measurements. T-test, Fisher's Exact tests and logistic regression were used for statistical analysis.

Results: The SCCOR cohort included 220 obstructed participants: $44 \%$ female and $21.4 \%$ with elevated depressive symptoms. GOLD staging distribution was predominantly stage I and II. The COPDGene cohort included 745 obstructed participants: 44\% female and $13.0 \%$ with elevated depressive symptoms. GOLD distribution was predominantly stage II and III. In the SCCOR cohort, correlation between IL-6 and depressive symptoms trended toward significance $(\mathrm{p}=0.08)$. Multivariable modeling adjusted for $\mathrm{FEV}_{1}$, age, gender and medical comorbidities showed a significant association $(\mathrm{OR}=1.70,95 \% \mathrm{CI}=1.08-2.69)$. IL-6 was significantly associated with elevated depressive symptoms in COPDGene in both univariate $(\mathrm{p}=0.001)$ and multivariable modeling $(\mathrm{OR}=1.52,95 \% \mathrm{CI}=1.13-2.04)$.

Conclusion: Elevated plasma IL-6 levels are associated with depressive symptoms in individuals with COPD independent of airflow limitation and comorbid risk factors for depression. Our results suggest that systemic inflammation may play a significant and possibly bidirectional role in depression associated with COPD.

Keywords: IL-6, depression, beck depression inventory, BDI, HADS, TNF-a, CRP, SCCOR, COPDGene

\section{Introduction}

Chronic Obstructive Pulmonary Disease (COPD) is a common lung disease contributing to significant worldwide morbidity and mortality. ${ }^{1}$ The complex pathogenesis of COPD includes airway inflammation that ultimately results in airway obstruction. $^{2}$ Moreover, COPD has strong associations with a host of comorbidities including depression. $^{2-6}$ The estimated global prevalence of depression is less than $10 \%$; however, in stable COPD patients the prevalence of depression is $6-42 \%{ }^{7-12}$ Depression in COPD has been associated with worse patient reported outcomes, poor adherence to medical therapy and increased mortality. ${ }^{13-19}$

Prior studies have documented that patients with COPD have elevated systemic inflammatory markers including C-reactive protein (CRP), interleukin-6 (IL-6), 
tumor necrosis factor-alpha (TNF- $\alpha$ ), activated leukocytes and fibrinogen. ${ }^{20-28}$ Likewise, recent literature has shown that adults with major depressive disorder have associated elevations of IL-1ß, IL-6 and CRP. ${ }^{29-36}$ A link between systemic inflammation and depression in individuals with COPD has not been conclusively established.

$\mathrm{Lu}$ et al found that severity of airflow limitation defined as decreased $\mathrm{FEV}_{1} \%$ predicted and $\mathrm{FEV}_{1} / \mathrm{FVC}$ was associated with both inflammation (ie, IL-6, CRP) and depressive symptoms. ${ }^{24}$ Conversely, several studies have found no clear association between IL-6 and depression in the setting of COPD. Hanania et al found no correlation between IL-6 and depression scores in a cohort of individuals with moderate to severe COPD. ${ }^{37}$ Likewise, Janssen et al described no correlation between persistent systemic inflammation and symptoms of depression at baseline or 36 months. $^{28}$

In summary, the pathogenesis of COPD and depression both include systemic inflammation; however, evidence of the association between systemic inflammation and depression in COPD remains variable and lacks reproducibility. Therefore, we sought to (1) analyze the relationship between inflammatory biomarkers and depressive symptoms in a small cohort of obstructed individuals and (2) validate our findings with a second large prospective COPD cohort. We hypothesized that obstructed individuals with elevated systemic inflammatory biomarkers will have increased depressive symptoms. The abstract of this paper was presented at the American Thoracic Society Conference as a poster presentation with interim findings. The poster's abstract was published in "Poster Abstracts" in ATS Journals: https://www.atsjournals.org/doi/abs/10. $\underline{1164 / \text { ajrccm-conference.2019.199.1_MeetingAbstracts. }}$ A4526.

\section{Methods}

\section{Study Population}

The COPD Specialized Center for Clinically Oriented Research (SCCOR) study is a single center, prospective observational cohort study of current and former smokers at the University of Pittsburgh. The study enrolled 727 participants between June 2008 and June 2010. Follow-up visits were completed at two, six, eight and ten years. Primary inclusion criteria required individuals to be at least 40 years old with a minimum of 10 pack-years tobacco smoking history at enrollment. Exclusion criteria consisted of evidence of another significant pulmonary diagnosis, history of lung cancer and obesity with a BMI greater than $34 \mathrm{~kg} / \mathrm{m}^{2}$. Additional details of the SCCOR cohort's selection criteria have been described previously. $^{38}$

The COPD Genetic Epidemiology (COPDGene) study is a multicenter prospective observational cohort study of current and former smokers designed to examine genetic factors associated with COPD. Over 10,000 participants were enrolled between January 2008 and June 2011 at 21 clinical centers. Primary inclusion criteria were age of 45 to 80 years old at enrollment and at least a 10 pack-year smoking history. Exclusion criteria consisted of alternate pulmonary diagnosis (exception of asthma) and lung cancer. The complete inclusion and exclusion criteria are listed in a previous COPDGene study publication. ${ }^{39}$

We prospectively screened inflammatory biomarkers including CRP, TNF- $\alpha$ and IL- 6 in the SCCOR cohort. A sample of 745 participants in the COPDGene cohort was then identified based on the presence of FEV1/FVC $<0.7$, IL-6 serum sample availability and Hospital Anxiety and Depression Scale Depression subscale (HADS-D) availability.

Both studies were conducted in accordance with the Declaration of Helsinki and good clinical practice guidelines. The SCCOR study was approved by the University of Pittsburgh ethics and review board. The COPDGene study was approved by the National Jewish Health, University of Colorado-Denver and each COPDGene site's ethics and review boards. All participants gave written informed consent for both the SCCOR and COPDGene cohort.

\section{Clinical Data and Definitions}

In both cohorts, COPD was defined as post bronchodilator spirometry $\mathrm{FEV}_{1} / \mathrm{FVC}$ less than 0.70 and categorized by the Global Initiative for Chronic Obstructive Lung Disease (GOLD) guidelines ${ }^{40}$. In addition to severity of depressive symptoms and biomarker measures, both the COPDGene and SCCOR cohorts collected pre- and postbronchodilator spirometry, chest computed tomography (CT) scans, St. George Respiratory Questionnaires (SGRQ) for evaluation of COPD-related symptoms, 6-minute walk distance (6MWD) in COPDGene, shuttle walk distance (SWD) in SCCOR and blood samples. Full details about SCCOR and COPDGene clinical data collection have been previously described. ${ }^{38,39}$ 


\section{Depressive Symptom Measurements}

Participants in each cohort were categorized into two groups, one with elevated depressive symptoms and one without elevated depressive symptoms. Depressive symptoms were measured at the baseline visit in both cohorts. Participants in the SCCOR cohort answered the Beck Depression Inventory-II (BDI-II) which is a 21 -item questionnaire with each item rated on a four-point scale ranging from no symptoms (0) to maximum symptoms (3). Total scoring ranges from 0 to 63 points. The BDI-II has satisfactory validity, sensitivity and specificity. ${ }^{41,42}$ The presence of current depression was defined as a BDI-II score of greater than 9, which was chosen to include participants with pre-clinical, mild, moderate and severe symptoms of depression. ${ }^{43}$ This was a threshold defined a-priori as a clinical cutoff to notify clinicians within the study prior to any analyses and has precedent in the literature. ${ }^{43}$ The BDI-II is validated in chronic inflammatory diseases, ${ }^{44}$ and has been shown to have consistent inter-prevalence with the HADS total score. ${ }^{45}$ Depression was assessed in COPDGene participants with the HADSD questionnaire. HADS-D is a 7-item depression subset questionnaire to the HADS total which includes 7 of each anxiety and depression questions. Symptoms are rated on a 4-point severity scale with 0 being no symptoms and 4 representing severe symptoms. The presence of depression was defined a HADS-D score of greater than 7. The HADS-D has satisfactory validity and specificity ${ }^{46}$ and has been validated in COPD patients. ${ }^{45,47}$ All participants reported medication use including antidepressants.

\section{Biomarker Measurements}

In the SCCOR cohort, baseline morning blood samples were collected in the fasting state and plasma samples were analyzed for thirty-three chemokines and growth factors, including those with a known link to COPD, such as TNF- $\alpha$, CRP and IL-6. IL-6 and TNF- $\alpha$ were measured using ultra-sensitivity ELESA kits (Invitrogen, Carlsbad, CA) and CRP was analyzed using MSD V-PLEX Vascular Injury Panel 2 Human Kit (MSD, Rockville, MD). For COPDGene, 114 blood biomarkers with links to COPD were collected at baseline in a nonfasting state. The biomarkers were evaluated using a 13panel multiplex assay (Myriad-RBM, Austin, TX). All assays and kits were used according to manufacturers' instructions. All samples were above the detection level for each measured biomarker. Additional details on the description of methods of the multiplex assay have been previously described. ${ }^{48,49}$

\section{Statistical Analysis}

The primary exposure and outcome investigated were inflammatory biomarkers and depressive symptoms, respectively. Inflammatory markers that have been previously associated with COPD and depression were selected for initial analysis, including TNF- $\alpha$, CRP and IL-6. Depressive symptoms were defined as a binary variable, based on questionnaire scores. Additional independent variables were $\mathrm{FEV}_{1}, \mathrm{FEV}_{1} / \mathrm{FVC}$, SGRQ, current smoking and exercise capacity (measured by 6MWD and SWD). T-test with best normal transformation was used for continuous variables and Fisher's Exact test was performed for categorical variables to compare participants with elevated depressive symptoms to those without elevated depressive symptoms. Because IL-6, CRP and TNF- $\alpha$ were not normally distributed, they were transformed by natural log for statistical analyses.

A total of six stepwise multivariable logistic regression models (three in each cohort) were used to assess the association between covariates and independent variables and elevated depressive symptoms entered as the dependent variable. Because IL-6 is colinear with severity of obstruction, we adjusted for $\mathrm{FEV}_{1} / \mathrm{FVC}$ as a continuous variable to determine whether inflammation was independently associated with depression. The first model was adjusted for demographic factors only including: age, gender and BMI. The second model was also adjusted for additional medical comorbidities that may be associated with depression including: history of stroke, diabetes and cancer. Heart failure was not included in the second model due to multicollinearity. Diabetes was not included for SCCOR because of low prevalence and multicollinearity. The third model was further adjusted for smoking status and $\mathrm{FEV}_{1} / \mathrm{FVC}$, confounders of general depression and depression related to COPD. ${ }^{50} \mathrm{P}$-value required for entry and retention in the model was $<0.05$. Participants with missing data were excluded from our analysis. All analyses were performed in STATA 16.2 (StataCorp, College Station, TX).

\section{Results}

\section{Participant Baseline Characteristics}

We examined 220 participants with $\mathrm{FEV}_{1} / \mathrm{FVC}<0.7$ in the SCCOR cohort and 745 participants in the COPDGene cohort. Participants in both cohorts were of similar age and 
BMI (Table 1). At baseline, $21 \%$ of the SCCOR cohort and $13 \%$ of the COPDGene cohort surpassed the threshold for depressive symptoms based on the BDI-II ( $>9)$ and HADS$\mathrm{D}(>7)$, respectively. Patients with depressive symptoms in SCCOR were divided evenly between male and female. In COPDGene, more males had depressive symptoms than females. Current smokers had higher incidence of depressive symptoms in both cohorts. The majority of the SCCOR participants were GOLD stage 1 and 2 while the majority of the COPDGene cohort was GOLD stage 2 and 3 (Table 1). Incidence of stroke, heart failure, diabetes and cancer was higher in participants without depressive symptoms in both SCCOR and COPDGene (Table S1).

\section{Determinants of Depression in Participants with COPD (Univariable Modeling)}

Univariate analysis at baseline revealed significant relationships between depressive symptoms and patient characteristics, COPD-related symptoms and biomarker measures. The depressed groups in each cohort shared decreased $\mathrm{FEV}_{1} / \mathrm{FVC}$, symptom burden and current smoking status. The depressed group in COPDGene notably demonstrated associations between younger age and decreased 6MWD. In SCCOR, a trend towards increased IL-6 was seen in participants with depressive symptoms that did not reach the level of statistical significance $(p=$ 0.08). In COPDGene, IL-6 was significantly elevated $(\mathrm{p}=0.001)$ in participants with depressive symptoms (Table 1). TNF- $\alpha$ and CRP were not associated with depressive symptoms in the SCCOR cohort (Table S2) and therefore were not analyzed in the COPDGene cohort.

\section{Determinants of Depression in Participants with COPD (Multivariable Modeling)}

To explore the determinants of depression in COPD, a parsimonious multivariable logistic regression model was performed. The final model included the following covariates: age, gender, BMI, cancer history, stroke history, diabetes,

Table I Baseline Characteristics Stratified by Depressive Symptoms

\begin{tabular}{|c|c|c|c|c|c|c|}
\hline & \multicolumn{3}{|c|}{ SCCOR $(\mathrm{N}=220)$} & \multicolumn{3}{|c|}{ COPDGene $(\mathrm{N}=745)$} \\
\hline & \multicolumn{6}{|c|}{ Depressive Symptoms } \\
\hline & Minimal & Elevated & p-value & Minimal & Elevated & p-value \\
\hline $\mathrm{N}$ & 173 & 47 & & 648 & 97 & \\
\hline Age, mean (SD) & $67.2(6.7)$ & $65.5(6.5)$ & 0.12 & $69.6(8.2)$ & $66.7(8.5)$ & 0.002 \\
\hline Gender & & & 0.51 & & & 0.19 \\
\hline Male & 99 (57.2\%) & $24(5 \mathrm{I} .1 \%)$ & & 359 (55.4\%) & 61 (62.9\%) & \\
\hline Female & $74(42.8 \%)$ & $23(48.9 \%)$ & & $289(44.6 \%)$ & $36(37.1 \%)$ & \\
\hline BMI, mean (SD) & $27.8(4.2)$ & 26.5 & 0.04 & $26.2(4.0)$ & $26.5(4.1)$ & 0.53 \\
\hline Active Smokers & $66(38.2 \%)$ & 27 (57.4\%) & 0.02 & 187 (28.9\%) & 40 (4I.2\%) & 0.02 \\
\hline BDI-II, median (IQR) & $4.0(1.0,6.0)$ & $14.0(11.0,16.0)$ & $<0.001$ & & & \\
\hline HADS-D, median (IQR) & - & - & & $2.0(1.0,4.0)$ & $9.0(8.0,11.0)$ & $<0.001$ \\
\hline $\mathrm{FEV}_{1} / \mathrm{FVC}$, mean (SD) & $58.1(10.2)$ & $53.8(10.6)$ & 0.01 & $53.0(12.5)$ & $48.3(13.5)$ & 0.002 \\
\hline $\mathrm{FEV}, \mathrm{PP}$, mean $(\mathrm{SD})$ & $71.2(18.1)$ & $66.0(18.4)$ & 0.09 & $61.8(23.0)$ & $52.4(23.2)$ & $<0.001$ \\
\hline SGRQ, mean (SD) & $21.4(15.4)$ & $38.1(15.8)$ & $<0.001$ & $26.7(18.5)$ & $49.3(20.9)$ & $<0.001$ \\
\hline SWD in feet, mean (SD) & $394.8(139.5)$ & $372.7(126.6)$ & 0.31 & & & \\
\hline $6 \mathrm{MWD}$ in feet, mean (SD) & & & & $1254.2(408.0)$ & $1045.6(396.5)$ & $<0.001$ \\
\hline IL-6, median (IQR) & $1.3(0.9,2.1)$ & $2.0(1.0,2.6)$ & 0.08 & $1.9(1.2,2.8)$ & $2.4(1.5,3.9)$ & 0.001 \\
\hline Gold Stage & & & 0.01 & & & 0.002 \\
\hline 1 & 59 (34.1\%) & 8 (17.0\%) & & I58 (24.4\%) & II (II.3\%) & \\
\hline II & 88 (50.9\%) & 33 (70.2\%) & & $266(41.0 \%)$ & 39 (40.2\%) & \\
\hline III & 25 (14.5\%) & $4(8.5 \%)$ & & $162(25.0 \%)$ & 28 (28.9\%) & \\
\hline IV & I (0.6\%) & $2(4.3 \%)$ & & $62(9.6 \%)$ & $19(19.6 \%)$ & \\
\hline
\end{tabular}

Abbreviations: BMI, Body Mass Index; BDI-II, Beck Depression Inventory-II; HADS-D, Hospital Anxiety and Depression Score, Depression Subset; SGRQ, St. George's Respiratory Questionnaire; SWD, Shuttle Walk Distance; 6MWD, Six Minute Walk Distance. 
Table 2 The Association Between Depressive Symptoms and IL-6 Levels

\begin{tabular}{|l|c|c|c|c|}
\hline \multirow{2}{*}{} & \multicolumn{2}{|c|}{ SCCOR } & \multicolumn{2}{c|}{ COPDGene } \\
\cline { 2 - 5 } & OR (95\% Cl) & p-value & OR (95\% CI) & p-value \\
\hline Unadjusted* & $1.79(1.12-2.86)$ & 0.014 & $1.60(1.23-2.08)$ & $<0.001$ \\
Non-pulmonary confounders $t$ & $1.79(1.12-2.86)$ & 0.014 & $1.61(1.23-2.11)$ & 0.001 \\
Pulmonary and non-pulmonary confounders¥ & $1.70(1.07-2.68)$ & 0.024 & $1.52(1.13-2.04)$ & 0.006 \\
\hline
\end{tabular}

Notes: *Adjusted for gender, age, BMI. ${ }^{+}$Adjusted for gender, age, BMI, stroke, diabetes, cancer history; diabetes not included for SCCOR. ${ }^{\sharp}$ Adjusted for gender, age, BMI, stroke, diabetes, cancer history, smoking status, FEV $/$ FVC; diabetes not included for SCCOR.

smoking status and $\mathrm{FEV}_{1} / \mathrm{FVC}$. Increased IL-6 and FEV /FVC independently correlated with depressive symptoms in obstructed individuals in both SCCOR and COPDGene (Table 2). In SCCOR, active smoking independently correlated with depressive symptoms. In COPDGene, younger age also independently correlated with depressive symptoms.

\section{Discussion}

The findings from these two large prospective cohorts confirm that systemic inflammation, represented by IL-6, is associated with depressive symptoms in obstructive lung disease. We found that IL-6 was significantly associated with depressive symptoms in both the SCCOR and COPDGene cohorts even after adjustment for key demographic factors and medical comorbidities known to be associated with depression. The model was adjusted for demographics, traditional measures of COPD severity via spirometry and medical comorbidities associated with depression. Interestingly, the correlation persisted despite differences in severity of obstruction amongst the two cohorts.

Prior studies assessing the relationship between inflammation and depression in COPD have yielded variable results. $\mathrm{Lu}$ et al found that IL-6, CRP and depressive symptoms were independently associated with decreased $\mathrm{FEV}_{1} \%$ predicted and $\mathrm{FEV}_{1} / \mathrm{FVC}$ in a large Singaporean population. ${ }^{24}$ Although most participants did not meet the threshold for airflow obstruction, this study supports the relationship between inflammatory markers, particularly IL-6, and depressive symptoms.

Al-shair et al investigated the relationship of inflammatory markers and depressive symptoms in a small British cohort with moderate COPD. ${ }^{21}$ They did not find an association between IL- 6 and depression in COPD but did conclude that TNF- $\alpha$ is linked with depression. The small size of the population and patient characteristics may have influenced the results. In contrast, our study included a range of severities of COPD and was adjusted for determinants of both general depression and risk factors for COPD-related depression. Hanania et al found no association between inflammatory markers (CRP, CCL-18, IL-6, IL-8, TNF- $\alpha$ and fibrinogen) and depressive symptoms amongst severely obstructed participants in a large international cohort. ${ }^{37}$ Janssen et al described no correlation between persistent systemic inflammation and symptoms of depression in a small subsection of a severely obstructed international cohort. These cohorts may not be representative of a singular demographic as patients were recruited worldwide. Our study included well-characterized American cohorts with all degrees of obstruction which is more representative of the heterogeneity of COPD in the United States.

While inflammation in our study cohorts was significantly associated with depressive symptoms, it is unclear why previous literature has not consistently produced similar results. One proposed mechanism suggests that depressive symptoms in subjects with COPD may be differentially modulated by factors such as systemic inflammation and respiratory symptoms at different levels of airflow obstruction. At mild to moderate airflow obstruction, systemic inflammation may be the dominant contributor to depressive symptoms when there is a lower overall degree of respiratory limitation. In the populations with more severe airflow obstruction, persistent respiratory symptoms and activity limitation may drive depression independent of systemic inflammation. Alternatively, there may be a distinct endotype of individuals who are predisposed to producing higher levels of inflammatory biomarkers which subsequently mediate depression.

The link between inflammation, COPD and depression can be explained on a molecular level. IL-6 is increased as a result of systemic inflammation from COPD. The release of IL-6 induces systemic inflammatory pathways including the kynurenine pathway. ${ }^{20,32,33,51}$ This pathway determines homeostasis of serotonin by degrading its precursor, tryptophan. ${ }^{33}$ Serotonin is a key neurotransmitter mediating depression and systemic inflammation can result in pathologically low levels of serotonin. ${ }^{34}$ IL-6 elevation therefore has a plausible pathobiological link to depression. 
Our study has several potential limitations that deserve comment. First, we assessed depressive symptoms using questionnaire measures instead of clinical diagnostic interviews, the gold standard for DSM-IV diagnosis. The two questionnaires used (BDI-II and HADS) have only $80-90 \%$ sensitivity and $60-70 \%$ specificity when compared to clinical diagnostic interviews. ${ }^{41,42,46}$ Secondly, this study was a post-hoc analysis based on convenience sampling which introduces selection bias. Third, the prevalence of comorbidities in our study was low, preventing us from evaluating the impact of comorbidities on IL-6 and depression. In fact, the relationship was the inverse of what we expected likely due to the small number of participants with comorbidities. Lastly, we are limited in only making associations using these cohort studies and would need to examine prospective data to determine changes over time. Ultimately, a randomized controlled trial would be necessary to examine a causal relationship between inflammation and depressive symptoms in COPD.

The implications of our study are two-fold. Firstly, depression is a multi-faceted comorbidity of COPD which is affected by many different physiological, psychological and social factors. The relationship between depressive symptoms in COPD and inflammation measured by IL- 6 implies that inflammation may be a common mediator between depression and COPD and possibly even act synergistically. The correlation established through our study can be used as foundational evidence upon which to develop larger prospective trials and possibly interventional studies targeting IL-6 to treat depression.

\section{Conclusion}

We found that depressive symptoms in COPD are related to elevated baseline levels of IL-6 independent of obstruction in two large well-defined cohorts. These findings suggest that inflammation may be a mediator of depression in COPD. Further studies should be performed to better understand the etiology and causal relationship of depression in COPD.

\section{Abbreviations}

COPD, Chronic Obstructive Pulmonary Disease; CRP, C-reactive protein (CRP); IL-6, Interleukin-6; TNF- $\alpha$, Tumor necrosis factor alpha; $\mathrm{FEV}_{1}$, Forced Expiratory Volume in 1 Second; $\mathrm{FEV}_{1} / \mathrm{FVC}$, Forced Expiratory Volume in 1 Second Divided by Forced Vital Capacity; SCCOR, Specialized Center for Clinically Oriented Research; COPDGene, COPD Genetic Epidemiology; HADS-D, Hospital Anxiety and Depression Scale, Depression Subscale; GOLD, Global Initiative for Chronic
Obstructive Lung Disease; CT, Computed Tomography; SGRQ, Saint George's Respiratory Questionnaire; 6MWD, 6 Minute Walk Distance; SWD, Shuttle Walk Distance; BDIII, Beck Depression Inventory-II.

\section{Data Sharing Statement}

The data supporting our findings is available upon request with permission of the SCCOR and COPDGene groups. This article has an online data supplement.

\section{Acknowledgments}

The authors would like to thank the participants of the SCCOR and COPDGene cohorts.

\section{Author Contributions}

All authors made a significant contribution to the work reported, whether that is in the conception, study design, execution, acquisition of data, analysis and interpretation, or in all these areas; took part in drafting, revising or critically reviewing the article; gave final approval of the version to be published; have agreed on the journal to which the article has been submitted; and agreed to be accountable for all aspects of the work.

\section{Funding}

This study was supported by the National Institute of Health (NIH) National Heart, Lung and Blood Institute (NHLBI) grants: NIH-NHLBI P50 HL084948 and NIHNHLBI U01 HL089897.

\section{Disclosure}

The authors have no conflicts of interest for this work to disclose.

\section{References}

1. World Health Organization. The Global Burden of Disease: 2004 Update. World Health Organization; 2008.

2. von Leupoldt A, Fritzsche A, Trueba AF, Meuret AE, Ritz T. Behavioral medicine approaches to chronic obstructive pulmonary disease. Ann Behav Med. 2012;44(1):52-65. doi:10.1007/s12160012-9348-7

3. Maurer J, Rebbapragada V, Borson S, et al. Anxiety and depression in COPD: current understanding, unanswered questions, and research needs. Chest. 2008;134(4Suppl):43S-56S. doi:10.1378/chest.08-0342

4. Yohannes AM, Alexopoulos GS. Depression and anxiety in patients with COPD. Eur Respir Rev. 2014;23(133):345-349. doi:10.1183/ 09059180.00007813

5. Ulrik CS, Vijverberg S, Hanania NA, Diamant Z. Precision medicine and treatable traits in chronic airway diseases - where do we stand? Curr Opin Pulm Med. 2020;26(1):33-39. doi:10.1097/ MCP.0000000000000639 
6. Young AL, Bragman FJS, Rangelov B, et al. Disease progression modeling in chronic obstructive pulmonary disease. Am J Respir Crit Care Med. 2020;201(3):294-302. doi:10.1164/rccm.201908-1600OC

7. Zhang MW, Ho RC, Cheung MW, Fu E, Mak A. Prevalence of depressive symptoms in patients with chronic obstructive pulmonary disease: a systematic review, meta-analysis and meta-regression. Gen Hosp Psychiatry. 2011;33(3):217-223. doi:10.1016/j. genhosppsych.2011.03.009

8. Matte DL, Pizzichini MM, Hoepers AT, et al. Prevalence of depression in COPD: a systematic review and meta-analysis of controlled studies. Respir Med. 2016;117:154-161. doi:10.1016/j. rmed.2016.06.006

9. Steel Z, Marnane C, Iranpour C, et al. The global prevalence of common mental disorders: a systematic review and meta-analysis 1980-2013. Int J Epidemiol. 2014;43(2):476-493. doi:10.1093/ije/ dyu038

10. Demyttenaere K, Bruffaerts R, Posada-Villa J, et al. Prevalence, severity, and unmet need for treatment of mental disorders in the World Health Organization world mental health surveys. JAMA. 2004;291(21):2581-2590. doi:10.1001/jama.291.21.2581

11. Schneider C, Jick SS, Bothner U, Meier CR. COPD and the risk of depression. Chest. 2010;137(2):341-347. doi:10.1378/chest.09-0614

12. van Ede L, Yzermans CJ, Brouwer HJ. Prevalence of depression in patients with chronic obstructive pulmonary disease: a systematic review. Thorax. 1999;54(8):688-692. doi:10.1136/thx.54.8.688

13. Agustí A, Edwards LD, Rennard SI, et al. Persistent systemic inflammation is associated with poor clinical outcomes in COPD: a novel phenotype. PLoS One. 2012;7(5):e37483. doi:10.1371/journal. pone. 0037483

14. Iyer AS, Holm KE, Bhatt SP, et al. Symptoms of anxiety and depression and use of anxiolytic-hypnotics and antidepressants in current and former smokers with and without COPD - a cross sectional analysis of the COPDGene cohort. $J$ Psychosom Res. 2019;118:18-26. doi:10.1016/j.jpsychores.2019.01.002

15. Hilmarsen CW, Wilke S, Engan H, et al. Impact of symptoms of anxiety and depression on COPD assessment test scores. Eur Respir J. 2014;43(3):898-900. doi:10.1183/09031936.00163913

16. Dahlén I, Janson C. Anxiety and depression are related to the outcome of emergency treatment in patients with obstructive pulmonary disease. Chest. 2002;122(5):1633-1637. doi:10.1378/ chest.122.5.1633

17. Yohannes AM, Baldwin RC, Connolly MJ. Prevalence of sub-threshold depression in elderly patients with chronic obstructive pulmonary disease. Int J Geriatr Psychiatry. 2003;18(5):412-416. doi:10.1002/gps.851

18. Felker B, Katon W, Hedrick SC, et al. The association between depressive symptoms and health status in patients with chronic pulmonary disease. Gen Hosp Psychiatry. 2001;23(2):56-61. doi:10.1016/s0163-8343(01)00127-x

19. Mikkelsen RL, Middelboe T, Pisinger C, Stage KB. Anxiety and depression in patients with chronic obstructive pulmonary disease (COPD). A review. Nord $J$ Psychiatry. 2004;58(1):65-70. doi:10.1080/08039480310000824

20. Moermans C, Heinen V, Nguyen M, et al. Local and systemic cellular inflammation and cytokine release in chronic obstructive pulmonary disease. Cytokine. 2011;56(2):298-304. doi:10.1016/j. cyto.2011.07.010

21. Al-shair K, Kolsum U, Dockry R, Morris J, Singh D, Vestbo J. Biomarkers of systemic inflammation and depression and fatigue in moderate clinically stable COPD. Respir Res. 2011;12:3. doi:10.1186/1465-9921-12-3

22. Chen Y-WR, Leung JM, Sin DD, Eickelberg O. A systematic review of diagnostic biomarkers of COPD exacerbation. PLoS One. 2016;11 (7):e0158843. doi:10.1371/journal.pone.0158843
23. Wei J, Xiong XF, Lin YH, Zheng BX, Cheng DY. Association between serum interleukin- 6 concentrations and chronic obstructive pulmonary disease: a systematic review and meta-analysis. PeerJ. 2015;3:e1199. doi:10.7717/peerj.1199

24. Lu Y, Feng L, Nyunt MS, Yap KB, Ng TP. Systemic inflammation, depression and obstructive pulmonary function: a population-based study. Respir Res. 2013;14:53. doi:10.1186/1465-9921-14-53

25. Chang SS, Vaz Fragoso CA, Van Ness PH, Fried LP, Tinetti ME. Association between combined interleukin-6 and C-reactive protein levels and pulmonary function in older women: results from the women's health and aging studies I and II. J Am Geriatr Soc. 2011;59(1):113-119. doi:10.1111/j.1532-5415.2010.03203.x

26. Mannino DM, Ford ES, Redd SC. Obstructive and restrictive lung disease and markers of inflammation: data from the third national health and nutrition examination. Am J Med. 2003;114(9):758-762. doi:10.1016/S0002-9343(03)00185-2

27. de Torres JP, Cordoba-Lanus E, López-Aguilar C, et al. C-reactive protein levels and clinically important predictive outcomes in stable COPD patients. Eur Respir J. 2006;27(5):902-907. doi:10.1183/ 09031936.06 .00109605

28. Janssen DJ, Mullerova H, Agusti A, et al. Persistent systemic inflammation and symptoms of depression among patients with COPD in the ECLIPSE cohort. Respir Med. 2014;108(11):1647-1654. doi:10.1016/j.rmed.2014.07.013

29. Hodes GE, Ménard C, Russo SJ. Integrating interleukin-6 into depression diagnosis and treatment. Neurobiol Stress. 2016;4:15-22. doi:10.1016/j.ynstr.2016.03.003

30. Ting EY, Yang AC, Tsai SJ. Role of interleukin-6 in depressive disorder. Int $J$ Mol Sci. 2020;21(6):2194. doi:10.3390/ ijms21062194

31. Valkanova V, Ebmeier KP, Allan CL. CRP, IL-6 and depression: a systematic review and meta-analysis of longitudinal studies. J Affect Disord. 2013;150(3):736-744. doi:10.1016/j.jad.2013.06.004

32. Allison DJ, Ditor DS. The common inflammatory etiology of depression and cognitive impairment: a therapeutic target. $J$ Neuroinflammation. 2014;11(1):151. doi:10.1186/s12974-0140151-1

33. Miller AH, Raison CL. The role of inflammation in depression: from evolutionary imperative to modern treatment target. Nat Rev Immunol. 2016;16(1):22-34. doi:10.1038/nri.2015.5

34. Coppen A. The biochemistry of affective disorders. Br J Psychiatry. 1967;113(504):1237-1264. doi:10.1192/bjp.113.504.1237

35. Zalli A, Jovanova O, Hoogendijk WJ, Tiemeier H, Carvalho LA. Low-grade inflammation predicts persistence of depressive symptoms. Psychopharmacology. 2016;233(9):1669-1678. doi:10.1007/s00213-015-3919-9

36. Howren MB, Lamkin DM, Suls J. Associations of depression with C-reactive protein, IL-1, and IL-6: a meta-analysis. Psychosom Med. 2009;71(2):171-186. doi:10.1097/PSY.0b013e3181907c1b

37. Hanania NA, Müllerova H, Locantore NW, et al. Determinants of depression in the ECLIPSE chronic obstructive pulmonary disease cohort. Am J Respir Crit Care Med. 2011;183(5):604-611. doi:10.1164/rccm.201003-0472OC

38. Bon J, Fuhrman CR, Weissfeld JL, et al. Radiographic emphysema predicts low bone mineral density in a tobacco-exposed cohort. Am $J$ Respir Crit Care Med. 2011;183(7):885-890. doi:10.1164/ rccm.201004-0666OC

39. Regan EA, Hokanson JE, Murphy JR, et al. Genetic epidemiology of COPD (COPDGene) study design. COPD. 2010;7(1):32-43. doi:10.3109/15412550903499522

40. Vogelmeier CF, Criner GJ, Martinez FJ, et al. Global strategy for the diagnosis, management and prevention of chronic obstructive lung disease 2017 report: GOLD executive summary. Respirology. 2017;22(3):575-601. doi:10.1111/resp.13012 
41. Beck AT, Ward CH, Mendelson M, Mock J, Erbaugh J. An inventory for measuring depression. Arch Gen Psychiatry. 1961;4:561-571. doi:10.1001/archpsyc.1961.01710120031004

42. Aben I, Verhey F, Lousberg R, Lodder J, Honig A. Validity of the beck depression inventory, hospital anxiety and depression scale, SCL-90, and Hamilton depression rating scale as screening instruments for depression in stroke patients. Psychosomatics. 2002;43 (5):386-393. doi:10.1176/appi.psy.43.5.386

43. Reeves GM, Rohan KJ, Langenberg P, Snitker S, Postolache TT. Calibration of response and remission cut-points on the beck depression inventory-second edition for monitoring seasonal affective disorder treatment outcomes. J Affect Disord. 2012;138(1-2):123-127. doi:10.1016/j.jad.2011.12.003

44. Wang YP, Gorenstein C. Psychometric properties of the beck depression inventory-II: a comprehensive review. Braz J Psychiatry. 2013;35(4):416-431. doi:10.1590/1516-4446-2012-1048

45. Bock K, Bendstrup E, Hilberg O, Lokke A. Screening tools for evaluation of depression in Chronic Obstructive Pulmonary Disease (COPD). A systematic review. Eur Clin Respir J. 2017;4(1):1332931. doi:10.1080/20018525.2017.1332931

46. Zigmond AS, Snaith RP. The hospital anxiety and depression scale. Acta Psychiatr Scand. 1983;67(6):361-370. doi:10.1111/j.16000447.1983.tb09716.x
47. Dowson C, Laing R, Barraclough R, et al. The use of the Hospital Anxiety and Depression Scale (HADS) in patients with chronic obstructive pulmonary disease: a pilot study. $N$ Z Med J. 2001;114 (1141):447-449.

48. Carolan BJ, Hughes G, Morrow J, et al. The association of plasma biomarkers with computed tomography-assessed emphysema phenotypes. Respir Res. 2014;15(1):127. doi:10.1186/s12931-014$0127-9$

49. Sun W, Kechris K, Jacobson S, et al. Common genetic polymorphisms influence blood biomarker measurements in COPD. PLoS Genet. 2016;12(8):e1006011. doi:10.1371/journal.pgen.1006011

50. Tsai TY, Livneh H, Lu MC, Tsai PY, Chen PC, Sung FC. Increased risk and related factors of depression among patients with COPD: a population-based cohort study. BMC Public Health. 2013;13:976. doi:10.1186/1471-2458-13-976

51. Réus GZ, Jansen K, Titus S, Carvalho AF, Gabbay V, Quevedo J. Kynurenine pathway dysfunction in the pathophysiology and treatment of depression: evidences from animal and human studies. J Psychiatr Res. 2015;68:316-328. doi:10.1016/j.jpsychires.2015.05.007

\section{Publish your work in this journal}

The International Journal of COPD is an international, peer-reviewed journal of therapeutics and pharmacology focusing on concise rapid reporting of clinical studies and reviews in COPD. Special focus is given to the pathophysiological processes underlying the disease, intervention programs, patient focused education, and self management protocols. This journal is indexed on PubMed Central, MedLine and CAS. The manuscript management system is completely online and includes a very quick and fair peer-review system, which is all easy to use. Visit http://www.dovepress.com/testimonials.php to read real quotes from published authors. 